\title{
Substitution Reduced Health Work Force Syndrome: A Way Forward for Developing Countries
}

\section{Rahman $\mathbf{R}^{\star}$}

Institute of Health Economics, University of Dhaka, Bangladesh

\begin{abstract}
World Health Organization (WHO) state Worlds 57 countries suffered from shortage of doctors in term of health worker imbalance, maldistribution. The objective of this study is to assess does substitution can be carried out in the mainstream of health systems to reduce health workforce syndrome for developing countries. Six facilities (two union health family welfare center, two Upazilla health complex and two clinics from Non-Government Organizations) were taken purposively. A structured questioner addressing one hundred and eleven (111) variables for Essential service package was used for in-depth interviews and observation. A hypothetical framework was used to focus on substitution, crisis and policy implication. Study report reveals that in seven components of Essential Service Package most of the services provided by doctors in public health facilities were same services served by non-doctor health care providers in health care facilities operated by non-government organizations. Non-doctor health care providers are not allow to serve even for non-communicable disease screening. Research result reveal that non-doctor health care service providers skill and expertise regards maternal health care, child health care, adolescent care, nutritional care and care for other diseases are gold standard. Regard maternal health care services non-doctor health care service providers not able to serve and referred the cases of identify obstetric emergencies (ANC7), labour induction (ND4), episiotomy (ND7) and identify and manage obstetric emergencies (isolation or B/CEmonC), obstructed labour, pre/eclampsia, haemorrhage, pre-term labour, including administration of antenatal Corticosteroids (ND8) and complicated cases (ND9), identification and management of obstetric complications:, Hemorrhage, Puerperal infection/sepsis (PNC4). In respect to neonatal care non-doctor providers not cannot manage preterm or low birth weight (LBW) neonate (INC4), identification and management of sepsis (NCD5), identification and management of omphalitis (NCD6), identification and management of LBW babies (refer <1, 800 (NCD7), identification and management of neonatal jaundice (NCD8) and obs and neonatal emergencies. Among family planning services non-doctor provider at NGO clinic do not serve the services are advocacy and awareness development on PPFP and post-MR/PAC-FP (FP2), menstrual regulation(FP11), post abortion FP(FP12), post-partum FP (FP13), post MR-FP (FP14), management of contraceptive complications (FP15). $\mathrm{IMCl}$ and EPI were well covered by non-doctor health care service providers following book chart let. Non-doctor health care service providers well capable to serve the services of essential service package (ESP). If top-up training given to them and allow to served more services that presently not covered would able to covered and doctors were get to engage themselves to manage more complicated cases. A special cadre will create in the mainstream of health service systems that increase service range in public health sector and able to ensure universal health coverage.
\end{abstract}

Keywords: Doctor (medical graduate); Non-doctor health care provider (Paramedic); Substitution; Essential Service Package (ESP)

\section{Introduction}

Presently the developing world suffers due to the health "workforce crisis." The World Health Report 2006 uses this term to study the current scenario in the developing countries. Health for All by $2000 \mathrm{AD}$ was announced at Alma-Ata Declaration in 1978 by World Health Organization (WHO) [1]. Primary Health Care (PHC) having eight elements and five components was considered as comprehensive approach of Alma-Ata Declaration. Essential Service Package (ESP) was the approach of Primary Health Care. This ESP is provided in all tires of health care service delivery facilities. Doctors (Medical graduate) were engage to provide this service. With the epidemiological transition of diseases, life style changes and for other factors like violence, road, traffic accident the priority of disease pattern is changed. Response to specific health problems required specific expert. Many countries of the world do not have sufficient number of doctors. According to WHO approximately seventy-five countries of the world had less than 2.5 health worker per 1000 population which is the minimum required number of provider for deliver the basic health services. The countries of South-East Asia region of WHO facing several common health workforce related problems. To develop an effective, efficient and equitable health systems for meeting the goal of improve population health, health work force should be appropriate in relation to number, skill-mix and distribution with optimum competency and motivation.

For projection of provider requirement usually used 'Ratio method (population to personnel)', here it assume 1 doctor for 1000 population and 1 nurse per 500 population, 'Need method (for health services based on professional judgments)', 'Demand method for health services based on self-perceived needs, and 'Target method (for production of services to meet specified targets based on both needs and demand)'. None of the country in the planet select any specific method for ensure availability of doctors. According to World Health Report for achieve a target of $80 \%$ coverage of skill birth attendance and child immunization country should have less than 2.28 doctor, nurses and midwives per 1000 population.

Human resource planning is a critical activity in broader sectorial

*Corresponding author: Rahman R, Institute of Health Economics, University of Dhaka, Bangladesh, Tel: +8801716-643645; E-mail: rafia643645@gmail.com

Received August 23, 2017; Accepted October 13, 2017; Published October 20, 2017

Citation: Rahman R (2017) Substitution Reduced Health Work Force Syndrome: A Way Forward for Developing Countries. J Glob Econ 5: 264. doi: 10.4172/23754389.1000264

Copyright: @ 2017 Rahman R. This is an open-access article distributed under the terms of the Creative Commons Attribution License, which permits unrestricted use, distribution, and reproduction in any medium, provided the original author and source are credited. 
planning. Human resource planning for health care service delivery should not focused only on healthcare professionals like doctors and nurses, but need to incorporate community health workers, mid-level workers (paramedics). It will strength the primary healthcare systems to increase coverage and able to serve the basic health needs of societies [2]. Worldwide there were 59.8 million health worker. About two third of them (39.5 million) provide health services. Other one third (19.8 million) are management and support workers. Developing countries were faces an acute shortage of different cadres of healthcare workers, which is bound to escalate further because of the high population growth rate, inequitable distribution and out-migration.

To meet the service demand health care providers at different level work together in different manner with super mutual understanding and make the changes in health workforce. Multidisciplinary working, changes in skill, role enhancement, role enlargement and substitution of workers are the five changes occurred in the delivery of health care services over the decade.

In multidisciplinary working individuals from different professions gathering together to provide care. It act as whole system approach to care inclusion of social services staff, community workers and volunteers of primary care teams. Its aim to support people to keep well through exercise, healthy eating, reducing alcohol consumption, stopping smoking, breastfeeding and so on. Such services are also known as public health or health prevention initiatives.

Organizing groups of workers with different professional backgrounds, skills, grades, qualifications, expertise and experience in order to achieve optimal patient care done at changing skill mix. The concepts of staff mix and skill mix have been used as policy and management tools for developing the best combinations of skills across professions and organizations as well as at the individual level. Role enhancement involves expanding a group of workers skills so they can assume a wider range of responsibilities through innovative and non-traditional roles. By altering task, staff are offered opportunities for individual achievement and recognition. As staff are involved in tasks that increase their responsibility, they feel honored and get satisfaction. Role enlargement has been present in many initiatives to up skill practitioners to provide certain routine, easily trainable and low risk procedures to support integrated care such as monitoring vital signs, measuring blood glucose level, measuring peak expiratory flow rate, examining for breast lumps and providing advice on health promotion. Role substitution involves extending practice scopes by encouraging the workforce to work across and beyond traditional professional divides. In recent decades role substitution has blurred traditional professional boundaries. In the US physician assistants with a wide variety of backgrounds, including nursing and social care, have become an attractive option for expanding workforce capacity. In many countries several types of non-doctor qualified staff members have been used as substitutes for nurses or doctors. Other role substitution examples include training respiratory therapists to perform EEGs and medical technologists to perform certain radiological procedures. Role delegation involves transferring certain responsibilities or tasks from one grade to another by breaking down traditional job demarcations [3].

Health care is a labor incentive industry and the health workforce is the foundation of the health system. When there are too few of all types of health workers relatives to the population the condition is called worker shortage. Too small pipeline and or high exit rate is the cause of worker shortage. If the cadres of health workers emerging from training is ill suites to the health need of the population the condition is called worker imbalance. When health workforce is not located in accordance with disease burden the condition is called spatial misdistribution. For example rural: urban disparity. When more than two problem persist called Syndromes in health workforce. This syndrome in health workforce would able to reduce by adopting role substitution.

Shortages of skilled health workers are reported in many specific health care areas. For example, there is an estimated shortage of 1.18 million mental health professionals, including 55,000 psychiatrists, 628,000 nurses in mental health settings and 493,000 psychosocial care providers needed to treat mental disorders in 144 low- and middle income countries (Appendix 1).

A literature search was conducted through Google, using the keywords "human resources for health, health workforce crisis, changes in health workforce over the decade, health workforce syndrome, Bangladesh and developing countries." Below Table 1 showing the countries already adopting the role substitution for providing health care services by non-doctor health care providers instead of doctors.

Health is the ultimate output in health sector. Policy interest is directed toward the production and cost of intermediate output, health care. Theoretically substitution does not mean that the two inputs are equivalent but rather that output can be produced using more than one technique. According to Bangladesh Demography and Health Survey 2011 the population size is 152.5 million and density of population is $946 / \mathrm{sqm}$. Out of 53, 063 registered physicians (medical graduate) 43,537 doctors are available in the country. Among this $38 \%$ doctors working under Ministry of Health and Family welfare and 58\% doctors

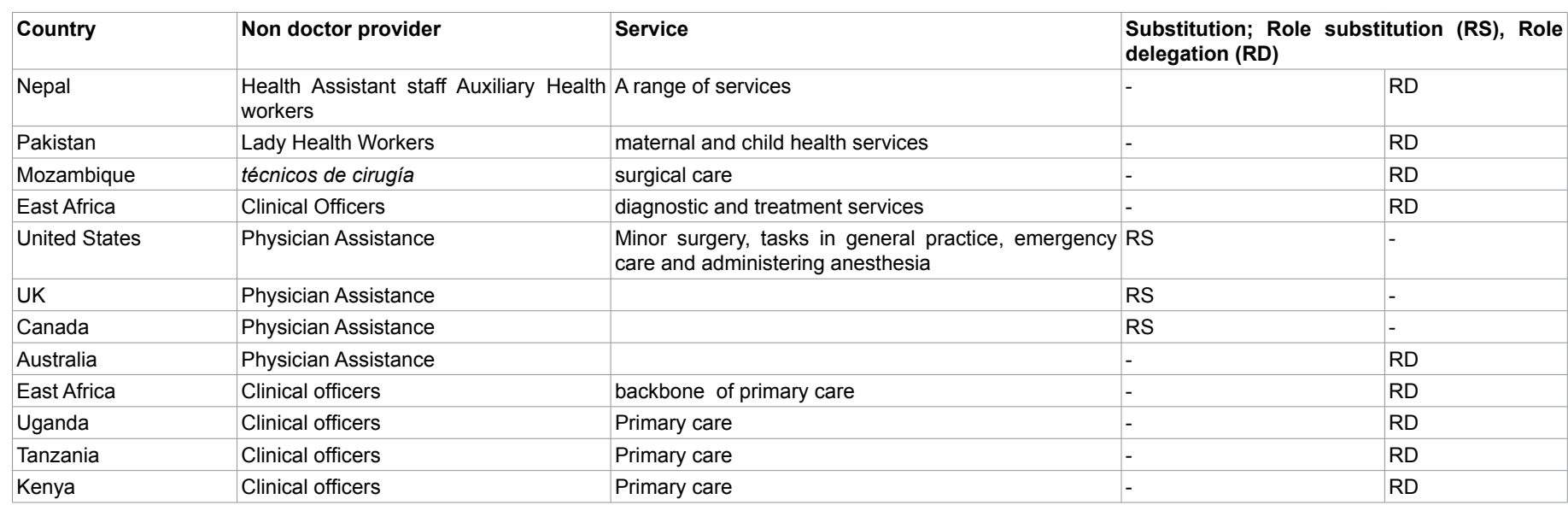

Table 1: Countries adopting the role substitution for providing health care services. 
working in private sectors. Number of registered diploma nurse is 26 , 899 among this 15023 available in country. Population per physician is 3269. Below table shows population wise health care services along with ESP and service provider. From ward to Upazilla level it is very essential to allow non-doctor health care service provider with top-up training, so they can extend their services as shown in Figure 1.

The density of doctor-nurses and dentists in Bangladesh is 7.7 per 10,000 population and constitute only about $5 \%$ of total health workforce. Bangladesh has a shortage of over 60,000 doctor and 280,000 nurses. Following ratio, need, and target and or demand method it is nearly impossible to produce the huge numbers of estimated doctors by the public and private sectors combined. At union and upazilla level if non-medical health care provider are allowed to provide the services like non-government organization operated clinic by incorporating new cadre. This approach able to reduce the shortage, misdistribution of doctors thus solve health work force syndrome.

The core services of ESP in Bangladesh are; i. maternal, neonatal, child and adolescent health care, ii. Family planning iii. Nutrition, iv. Communicable diseases, v. non-communicable diseases each component having sub components. Beside this according to Bangladesh Bureau of statistics pattern of disease burden are fever, pain, diarrhea and dysentery. So services for ESP and disease burden can provide by non-medical graduate health care providers with the establishment of a functional referral system to the higher level of facilities.

\section{Methods and Materials}

The objective of this study is to assess does substitution can be carried out in the mainstream of health systems to reduce health workforce syndrome for developing countries like Bangladesh.

Developing countries need to allow non-doctor health care provider in the mainstreams of health systems for provide health care services. After proper training non-doctor health care provider were able to provide health care service like doctors with professional perfection and technical skill for an extent of services. The clinics operated by NGO's providing services by non-doctor health care providers. Following developed countries and using the experience of NGO's clinic. A national strategies need to take in large scale to address the crisis in health workforce in term of address 'Health Workforce Syndrome' as shown in Figure 2.

Six facilities (two union health family welfare center, two Upazilla health complex and two clinics from NGO) were taken purposively to comparing services of doctor and non-doctor. A structured questioner addressing 111 variables for Essential service package was use for indepth interviews and observation. A hypothetical framework was use to focus on substitution, crisis and policy implication.

\section{Variables}

1. Maternal Care; Preconception care, ANC, Normal delivery, PNC

2. Neonatal care; Immediate newborn care, Newborn care after delivery, Obs And neonatal emergencies

3. Adolescent Health, Family planning

4. Nutrition; Child nutrition, maternal nutrition and Adolescent nutrition

5. Non communicable and other disease

6. Child health and EPI; Integrated management of child illness (IMCI), EPI

7. Other diseases; Eye care, Ear care, Dental care and skin care

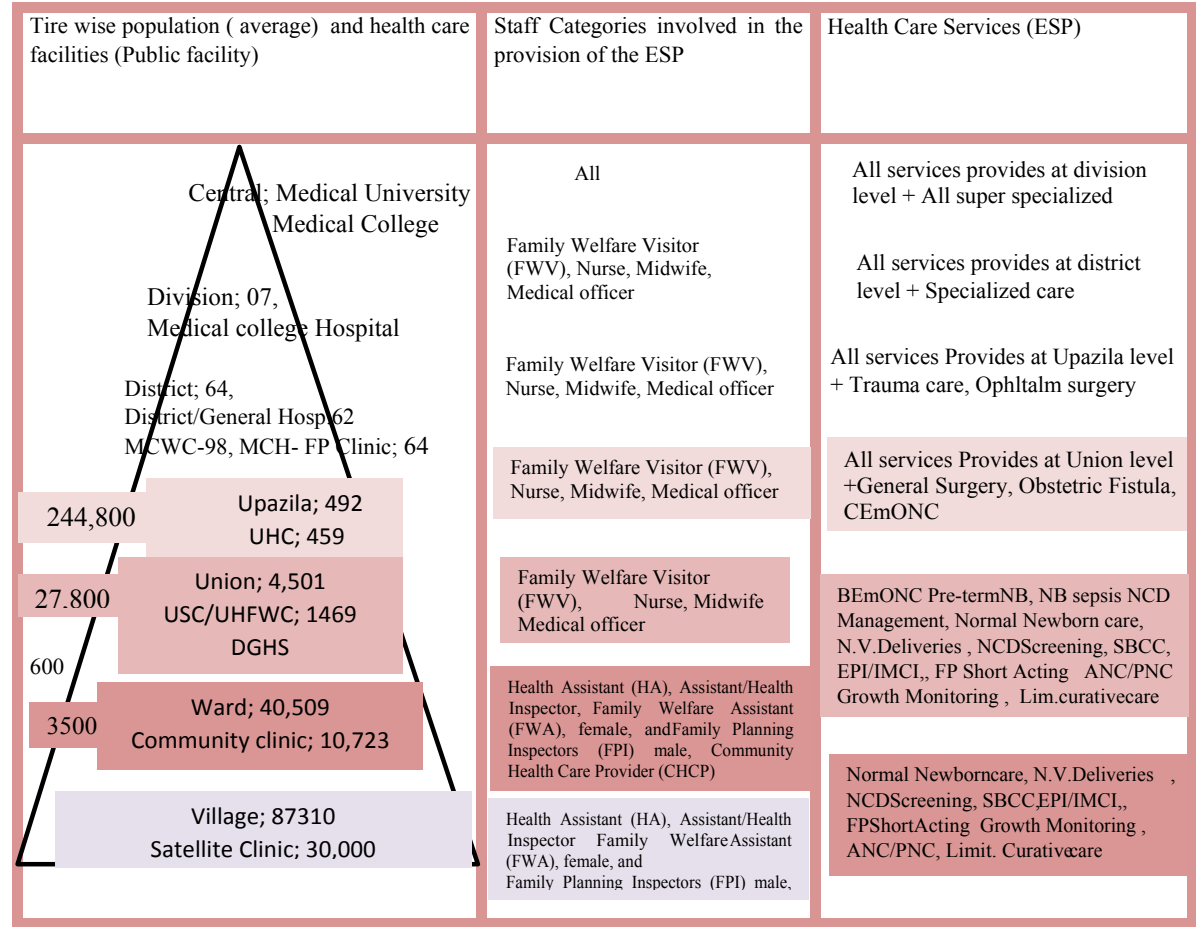

Figure 1: Illustration of health care services. 


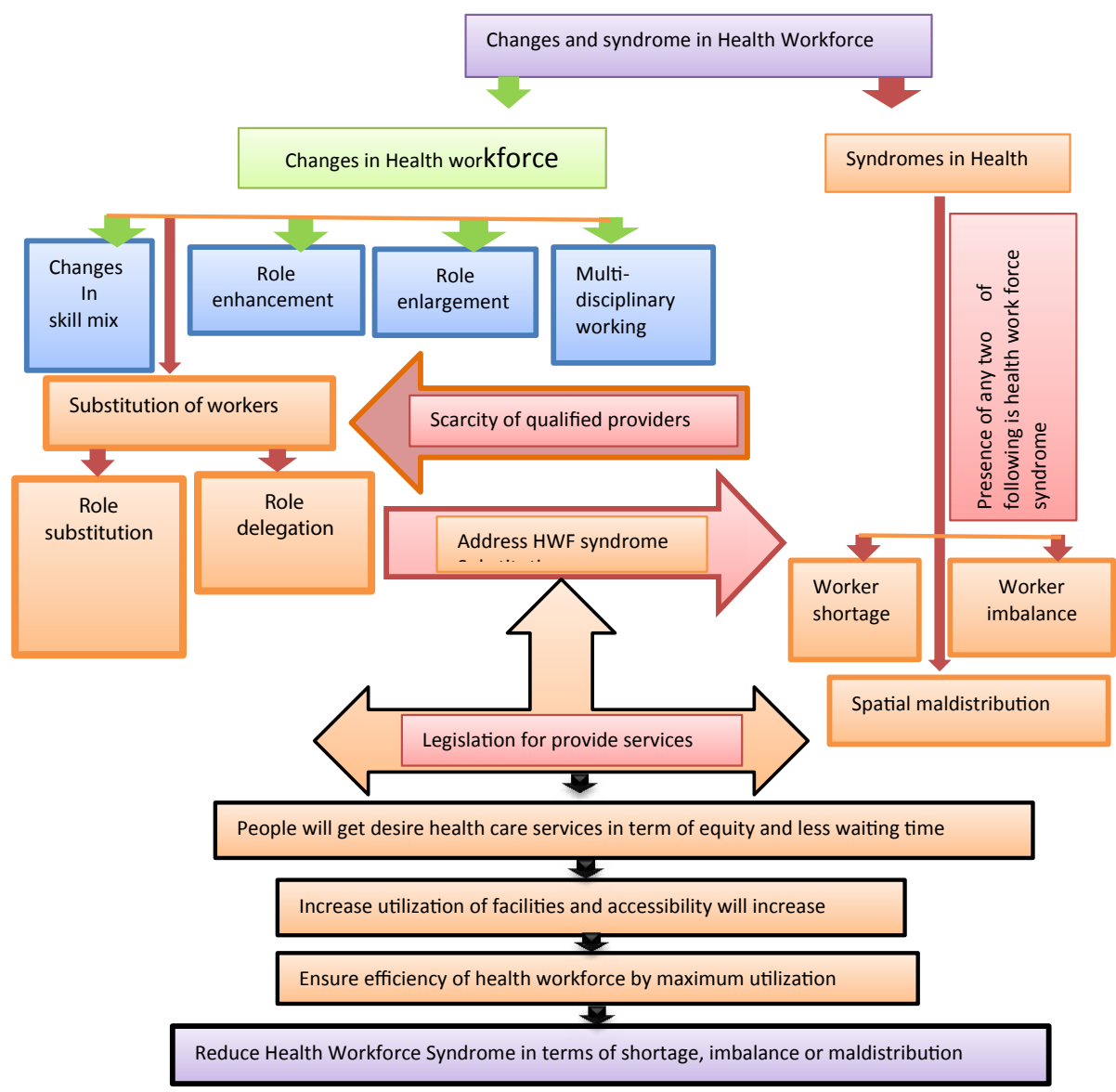

Figure 2: Hypothetical framework

8. Health care service (ESP) provider Doctor (medical graduate), Paramedic (non-doctor provider).

\section{Operational definition}

Non doctor provider: Individuals who are not doctors but have been completed three year course from recognized institute. Trained on "diagnose and treat common health problems, ESP, manage emergencies, refer appropriately and transfer the seriously ill or injured for further care. In this study paramedic is taken as non-doctor health care provider.

Role substitution involves extending practice scopes by encouraging the workforce to work across and beyond traditional professional divides. For examples include training respiratory therapists to perform EEGs and medical technologists to perform certain radiological procedures.

Role delegation: Role delegation involves transferring certain responsibilities or activities (service) from one grade to another by breaking down traditional job demarcations.

\section{Result and Discussion}

The study result reveals in all component of ESP service delivery provided by doctors at Union and Upazilla level health care facilities are delivered by non-doctor health care provider (paramedics) at clinics operated by NGO's which is shown in Figure 3A.

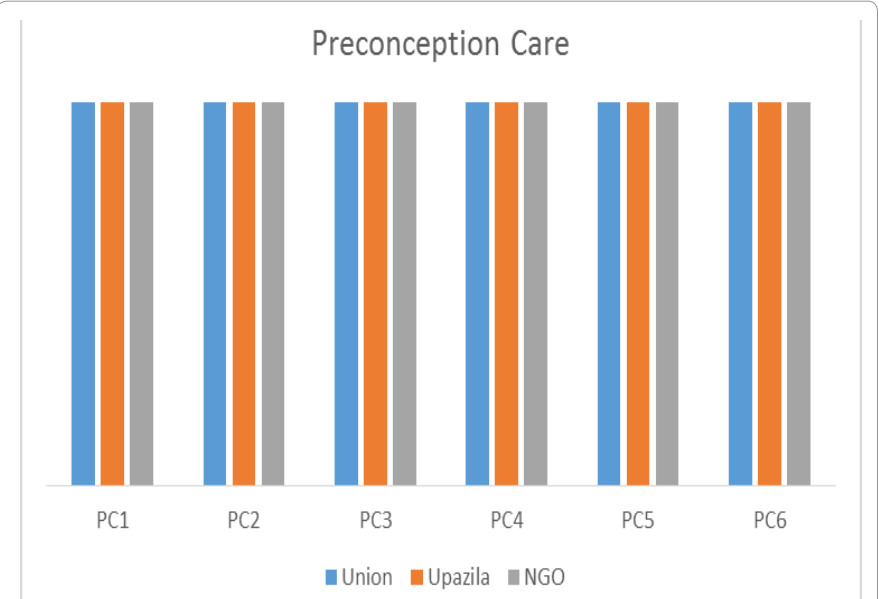

Figure 3a: Preconception care which is operated by NGO.

Maternal care covers the period starting when conception is planned to 42 days after delivery. Maternal care it covers the period starting when conception is planned to 42 days after delivery. Preconception care, antenatal, delivery, postnatal care included here. To assess pre-conception care six variables; promote health, family planning, nutrition, child survival and safe motherhood (P1), Screening for malnutrition (P2), Iron and Folic Acid supplementation (P3), Tetanus toxoid (P4), Family planning counseling (P5), identify 
RTI/STI (P6) was ask and observed at two lower tire of public facility and one NGO clinic. The bar graph shows that non-doctor provider at NGO clinic (paramedic), Family welfare visitor (FWV), Family welfare Assistant (FWA) at union level and doctor provider at Upazilla level providing equal level of care. Though FWA, FWV received only twenty one days training for serve this sub-component of ESP [4].

The skill of Antenatal care (ANC) is very crucial for both mother and pregnancy outcome. ANC consultation, clinical investigation and birth plan focused here. To compare non-doctor technical skill asked and observed for identification/diagnosis of pregnancy (ANC1), registration of pregnancy (ANC2), information and counselling on nutrition, complications of pregnancy, post-partum FP (ANC3), take clinical history (ANC4), Obstetric and fetal assessment, Maternal weight, BP measurement, Oedema, Fundal height, Foetal heartbeat (ANC5), identify and manage pregnancy complications:, Anaemia, Urinary tract infection (ANC6), Identify and manage obstetric emergencies (isolation or as part of B/CEmONC), Pre/ eclampsia, Ante-partum Haemorrhage, Abdominal pain, Premature rupture of membranes (ANC7), supply of Iron, Folic Acid and other micronutrients(ANC8), Tetanus Toxoid(ANC9), birth preparedness plan(Identification of SBA/referral hospital-setting aside money, identification of transportation means, identification of blood donor, clean kit for home delivery)-ANC10 [5]. Non-doctor provider at NGO and union can only identify obstetric emergencies not able to manage at all, they referred these cases. Obstetric emergencies can only managed by doctor at Upazilla level that is the first referral point of service delivery facility as shown in Figure 3B.

Delivery is the final outcome of pregnancy. The maternal death due to pregnancy occurs more at the time of delivery or for the complication of delivery. To measure skill of delivery ask and observe for Personal and obstetric history (ND1), Examination of foetal position- foetal heartbeat (ND2), monitor labour progression: Partograph (ND3), labour induction (ND4), conduct normal delivery (ND5), controlled cord traction (ND6), episiotomy (ND7), identify and manage obstetric emergencies (isolation or $\mathrm{B} / \mathrm{CEmonC}$ ), obstructed labour, pre/ eclampsia, haemorrhage, pre-term labour, including administration of antenatal corticosteroids (ND8), identify and referred complication (ND9). As Upazilla health complex (UHC) is the first referral center, they able to provide nine variable of the study. At NGO clinics have non-doctor health care provider they are not able to provide and they referred the cases of Labour induction (ND4), Episiotomy (ND7) and Identify and manage obstetric emergencies (isolation or B/CEmonC),

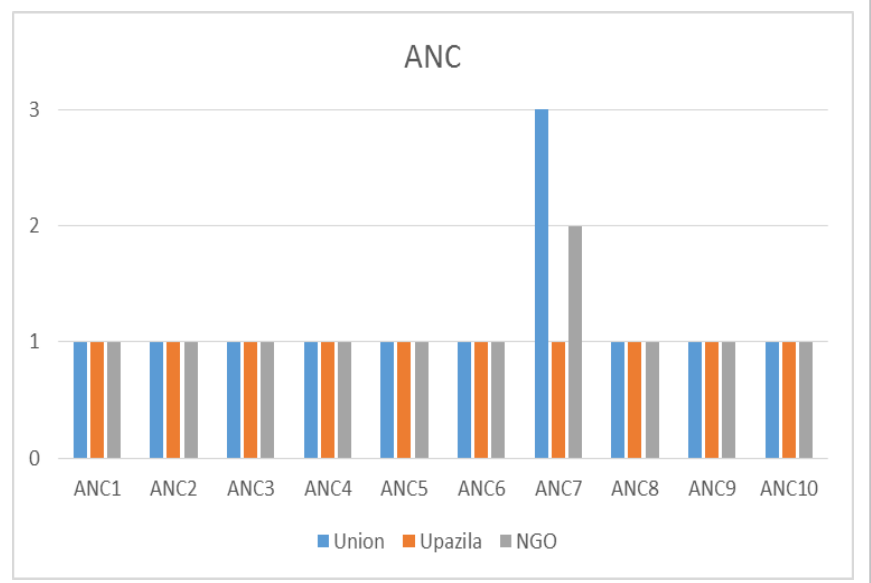

Figure 3b: The skill of antenatal care (ANC). obstructed labour, pre/eclampsia, haemorrhage, pre-term labour, including administration of Antenatal Corticosteroids (ND8). At union level, union health and family welfare center also referred the cases of labour induction (ND4), episiotomy (ND7) and identify and manage obstetric emergencies (isolation or B/CEmonC), obstructed labour, pre/eclampsia, haemorrhage, pre-term labour, including administration of antenatal Corticosteroids (ND8) and complicated cases (ND9) which is shown in Figure 4A.

Addressing ESP seven variables observed and asked for postnatal care variable used; counseling on postnatal care, breastfeeding (PNC1) taken Post-Natal clinical history (pain, fever, haemorrhage) (PNC2), identification and management of post-natal complications:, Anaemia, Puerperal psychosis (PNC3), identification and management of obstetric complications:, Haemorrhage, puerperal infection/sepsis (PNC4), supply of Iron and Folic Acid (PNC5), counselling and provision of FP methods (PNC6), identify and referred obs Fistula and prolapse (PNC7). Out of this seven non-doctor providers of NGO clinics and union health and family welfare center referred the cases of variable 4, identification and management of obstetric complications:, Haemorrhage, Puerperal infection/sepsis (PNC4). Union health and family welfare center also referred the cases of obs Fistula and prolapse (PNC7). These cases not report to NGO clinic usually as shown in Figure 4B.

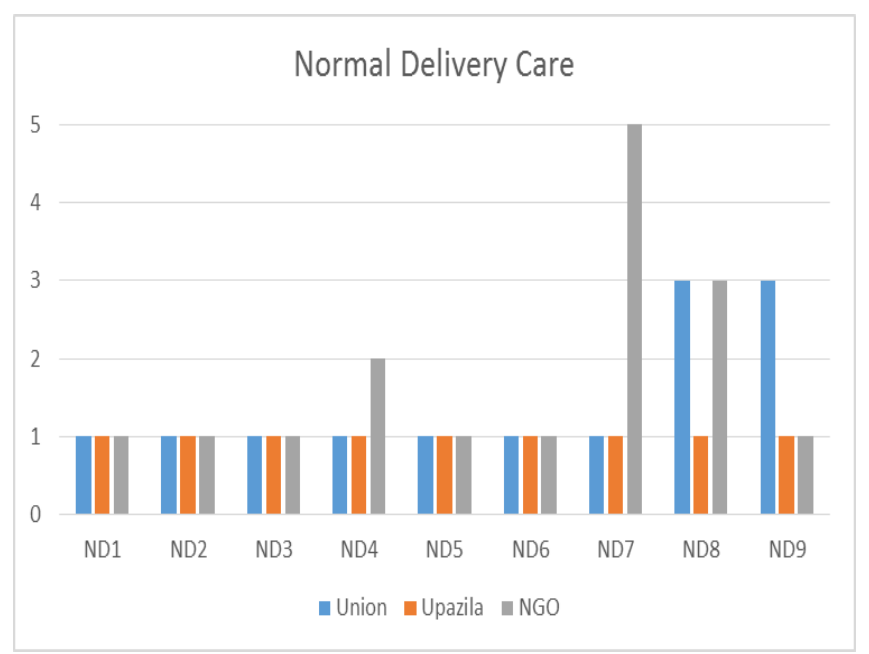

Figure 4a: Normal delivery care.

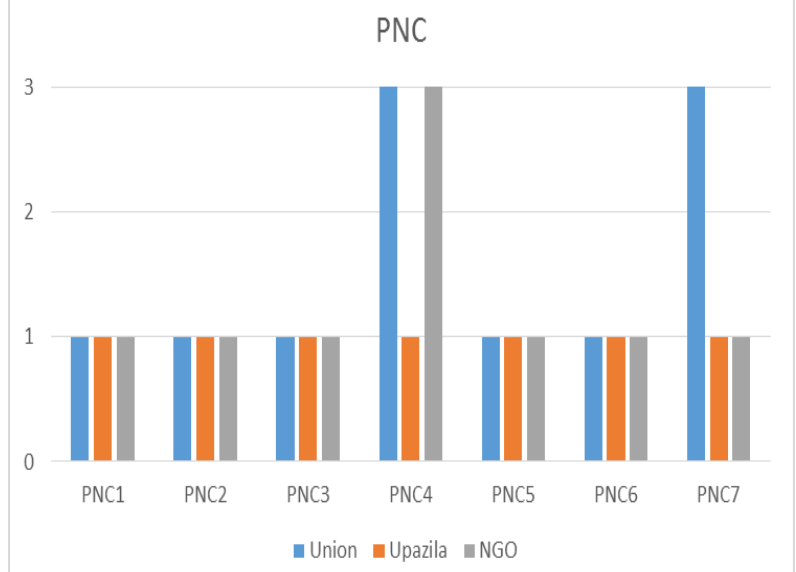

Figure 4b: Counseling on postnatal care. 


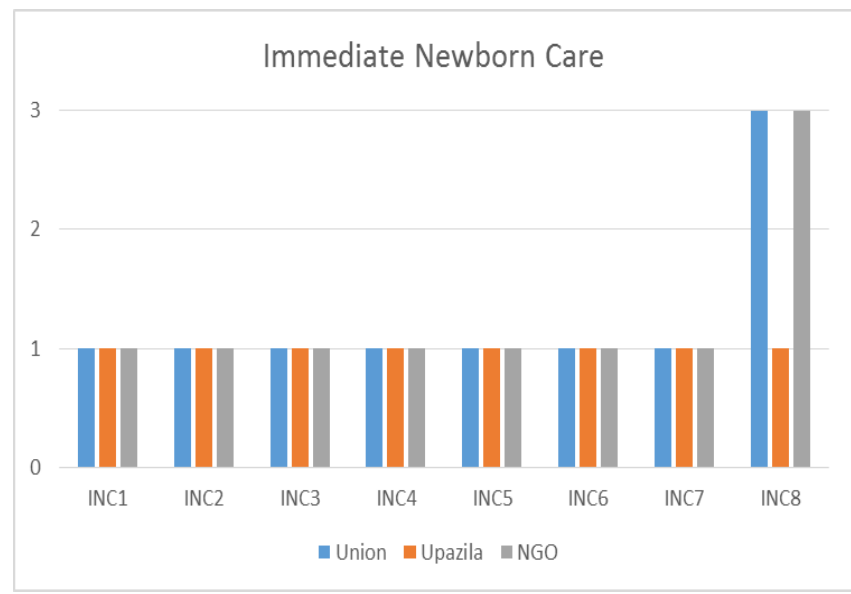

Figure 4c: Newborn care.

Regard immediate newborn care except special care of pre-term or LBW neonate, including Kangaroo Mother Care (INC8) all services promotion of essential newborn care practice and newborn danger signs for early care seeking (INC1), clean cord cutting and tying(INC2), single application of Chlorhexidine $7.1 \%$ to the cord (INC3), prevention and management of hypothermia, drying and wrapping, skin-toskin contact, delayed bathing (after $72 \mathrm{~h}$ ) (INC4), identification and management of breathing problems (digital stimulation, bag and mask resuscitation) (INC5), breastfeeding within one hour after delivery (INC6), prevention of newborn conjunctivitis (INC7) are maintain as gold standard by non-doctor providers [6].

Regarding newborn care after delivery eleven variables; counseling about breastfeeding, nutrition, immunization (NCD1), birth registration (NCD2), breastfeeding (NCD3), weighing, temperature management and cord care (NCD4), identification and management of sepsis (NCD5), identification and management of omphalitis (NCD6), identification and management of LBW babies (refer $<1800$ (NCD7), identification and management of neonatal jaundice (NCD8), identification and management of breastfeeding problems (NCD9), newborn immunizations (NCD10) was asked and observed. Among this NGO clinic referred the cases, identification and management of sepsis (NCD5), identification and management of omphalitis (NCD6), identification and management of LBW babies (refer $<1$, 800 (NCD7), identification and management of neonatal jaundice (NCD8) and Union health and family welfare center referred the cases- identification and management of neonatal jaundice (NCD8), identification and management of breastfeeding problems (NCD9) [7]. But Upazilla health complex able to serve all of services for newborn care after delivery shows in beside Figure 4C.

Obs and neonatal emergencies care include basic Obstetric and neonatal emergencies (all 7 services): parenteral antibiotics (ONE_ EPI1), parenteral anticonvulsants, (ONE_EPI2), parenteral uterotonics, (ONE_EPI3), manual removal of placenta, (ONE_EPI4), removal or retained products (manualvacuum aspiration) (ONE_EPI5), assisted vaginal delivery (ONE_EPI6), resuscitation of the newborn(ONE_ EPI7), BEmONC along with surgical capacity (caesarean section and others) (ONE_EPI8), blood transfusion (ONE_EPI9). The bar graph showing non-doctor health care provider at NGO clinic do not have the capacity to assisted vaginal delivery (ONE_EPI6), BEmONC along with surgical capacity (caesarean section and others) (ONE_EPI8).
Union health and family welfare center also not able to do BEmONC along with surgical capacity (caesarean section and others), (ONE_ EPI8). At the time of study there was no parenteral anticonvulsants (ONE_EPI2) at Upazilla health complex so they not able to provide as shown in Figure 5A.

The IMCI services mentioned in book chart let provided by UNICEF, WHO; counseling to parents on danger signs, nutrition of the sick child, identification of danger signs and referral: inability to drink or breastfeed, vomits everything, has/has had convulsions, lethargic or unconscious, management of Acute Respiratory Infection (ARI), cough/cold, fast breathing (pneumonia), severe pneumonia, wheeze, management of Diarrhoea: No dehydration, mild dehydration, severe dehydration, persistent diarrhea,, dysentery, management of fever and malaria (also included in CDC), simple fever, severe febrile disease, Lab diagnosis, Malaria, management of Ear problems:, acute ear infection, chronic ear infection, mastoiditis, counseling parents on immunization and adverse effect (EPI), follow-up to identify adverse effects, counseling to mothers regarding FP were well covered and manage by non-doctor health care service providers.

The Figure 5B is showing the skill of doctor and non-doctor health care providers for serve adolescent health care in terms of counseling on puberty, safe sexual behavior, prevention of early marriage, mental health, HIV/AIDS, substance abuse (AH1), screening for STI (AH2), syndrome management of STI (AH3), FP information and provision (AH4), adolescent Nutrition (Assessment, Iron/FA) (AH5) was same.

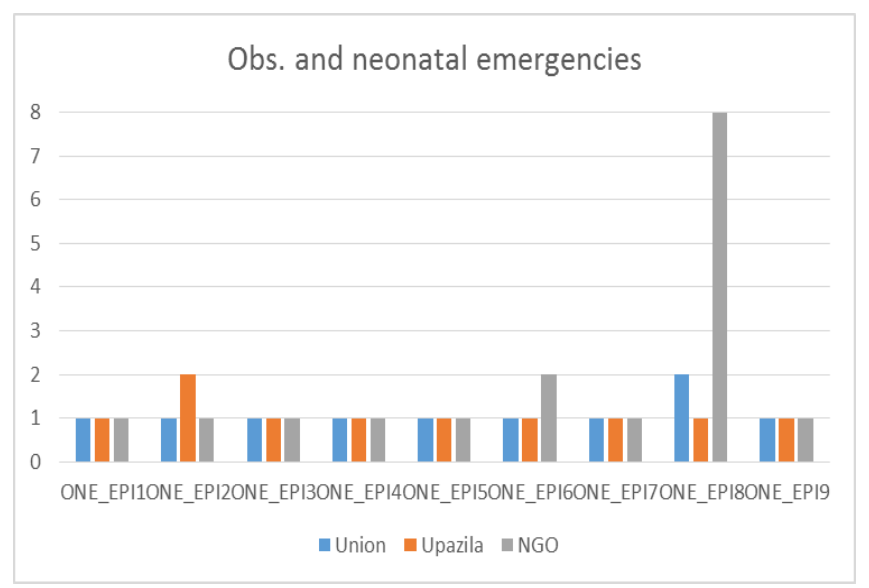

Figure 5a: Obstetric and neonatal emergencies.

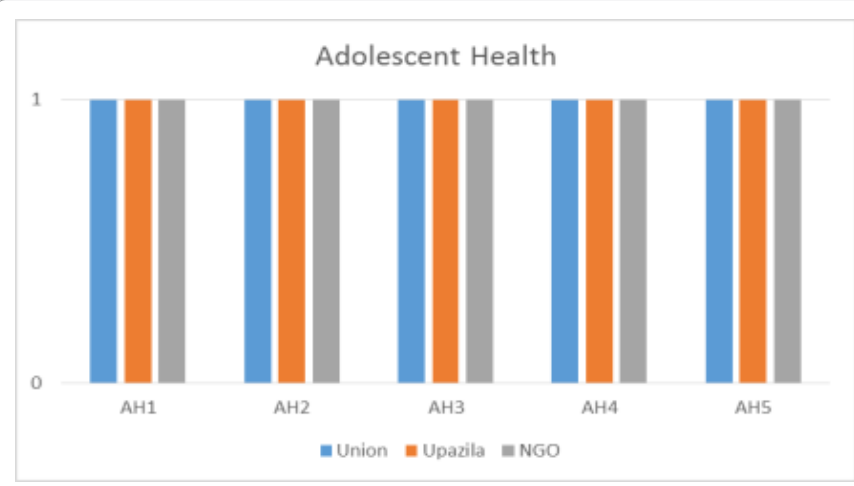

Figure 5b: Skill of doctor and non-doctor health care providers for serve adolescent health care. 
Variables used for assess family planning services was counseling on Birth Spacing, methods and adverse effects (FP1), advocacy and awareness development on PPFP and Post MR/PAC, FP (FP2), screening according to Medical Eligibility Criteria (MEC) for contraceptive use(FP3), pre-conception FP(FP4), condom (FP5), oral contraceptives (FP6), DMPA injection starting (FP7), intrauterine devices (FP8), implant (FP9), male and female sterilization (FP10), menstrual regulation (FP11), post abortion FP (FP12), post-partum FP (FP13), post MR-FP (FP14), management of contraceptive complications (FP15). The bar graph shows non-doctor provider at NGO clinic do not serve the services are advocacy and awareness development on PPFP and post MR/PAC-FP (FP2), menstrual regulation (FP11), post abortion FP(FP12), post-partum FP (FP13), post MR-FP (FP14), management of contraceptive complications (FP15). Union level not serves management of contraceptive complications (FP15) whereas Upazilla health complex able to serve all services regarding family planning as shown in Figure 5C.

Figure 6 showing regard child nutrition, counseling parents about breastfeeding and best feeding practices $(\mathrm{CN} 1)$, growth monitoring (CN2), community-level screening $(\mathrm{CN} 3)$, promotion of infant and young child feeding (IYCF): breastfeeding within an hour of birth, exclusive breastfeeding for 6 months, breastfeeding until 23 months of age, appropriate complementary feeding $(\mathrm{CN} 4)$, deworming $(\mathrm{CN} 5)$, micronutrient supplementation( $\mathrm{CN} 6)$, management of moderate acute malnutrition (CN7) was the asked and observed variables for assess child nutrition. Out of eight non-doctor providers of NGO's clinic not able to do Community-level screening (CN3) and management of moderate acute malnutrition (CN7).

The Figures 7-9, shows regarding the Variable for maternal and adolescent are Counseling on best practices during pregnancy (MN1),

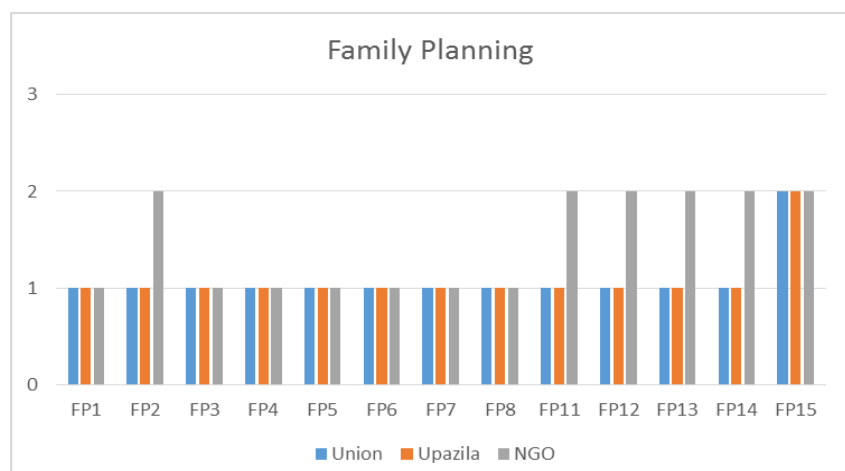

Figure 5c: Variables used for assess family planning services.

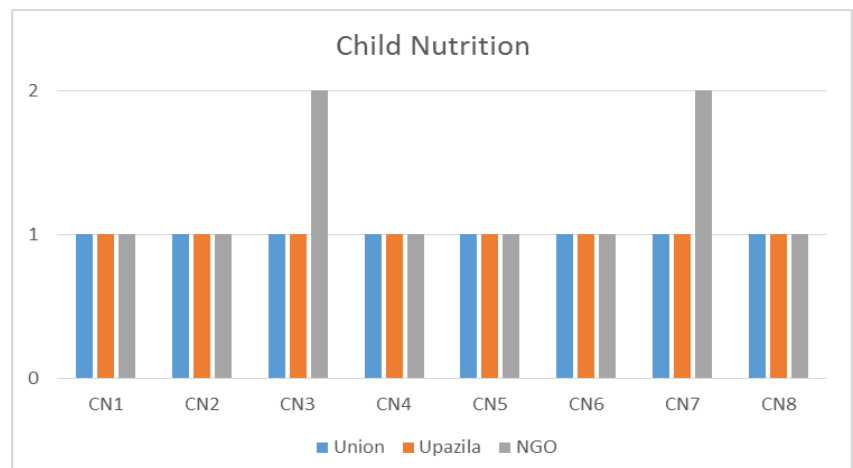

Figure 6: Child nutrition.

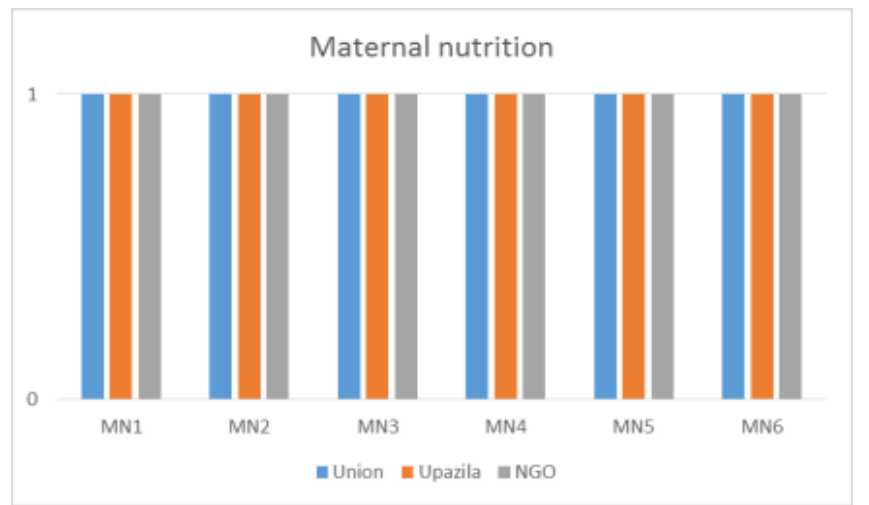

Figure 7: Variable for maternal Counseling.

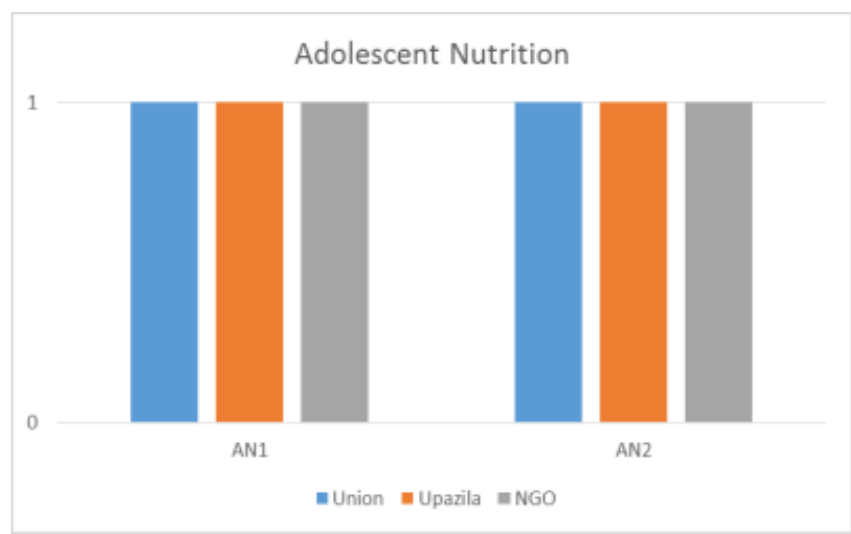

Figure 8: Variable for adolescent counseling.

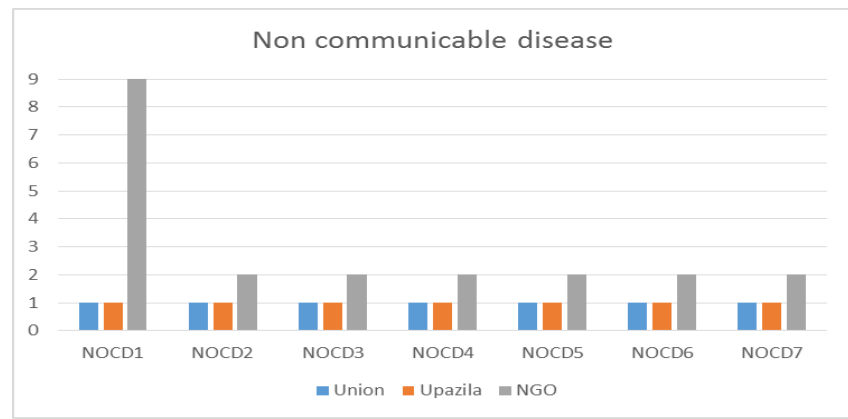

Figure 9: NGO clinic's regarding non-communicable diseases.

Assessment of nutritional status during ANC (MN2), Supplementation of Iron, Folic Acid, Calcium (MN3), Deworming (MN4), Management of anemia(MN5), Vit A in post-partum(MN6) and Assessment of nutritional status (AH1), Distribution of Iron/Folic Acid (AH2). In all level center non-doctor providers deliver their services like doctors at Upazilla health complex level [8].

Figure 10 is showing variables mentioned in ESP for noncommunicable disease was promote healthy lifestyle for HTN and other NCD control (NOCD1), identification and referral of long-term complications (DM) (NOCD2), screening for Risk Factors of CVD:, Family History of CVD/DM/kidney disease, High Blood Pressure, Smoking, Overweight, High Total Cholesterol, High Blood Sugar (NOCD3), counseling on screening of cervical and breast cancers 


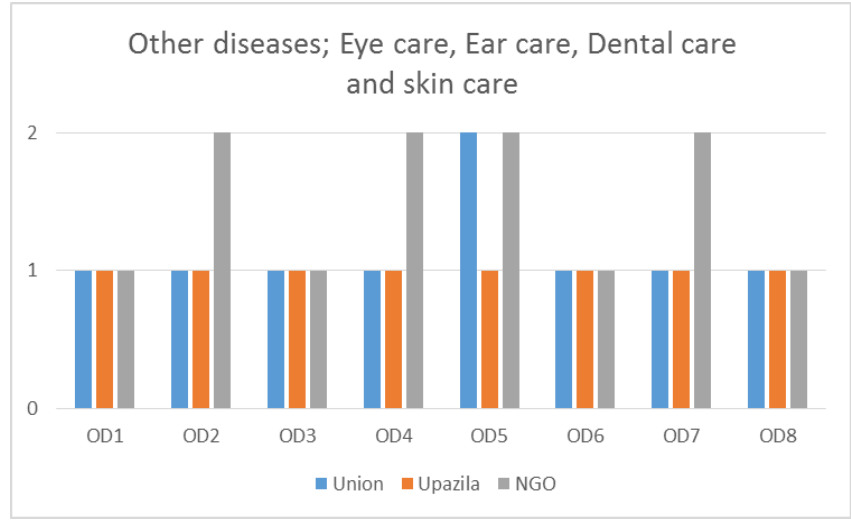

Figure 10: Other diseases observed for treatment of acute conjunctivitis.

(NOCD4), teaching of breast self-examination(NOCD5), counseling on identification and support to mental health cases, including fighting stigma and others(NOCD6), identification of signs of mental health conditions and referral, autism and neurodevelopmental disorders, epilepsy, common disorders:, depression,, psychosis,, anxiety and substance abuse(NOCD7). NGO clinic's non-doctor health care provider did not provide any care regarding non-communicable diseases.

To treat other diseases like eye care, ear care, dental care and skin care asked and observed for treatment of acute conjunctivitis (OD1), detection of cataract and visual impairment (OD2), awareness on prevention of hearing impairment (OD3), management of acute supportive otitis media (OD4), management of chronic otitis media (OD5), promotion of oral hygiene (OD6), treatment of common dental diseases (gingivitis, caries, etc.) (OD7), treatment of common skin diseases:, scabies, ringworm(OD8) [9]. Bar graph showing among eight variables non-doctor referred cataract and visual impairment (OD2) cases, management of acute supportive otitis media (OD4), management of chronic otitis media(OD5), and for treatment of common dental diseases (gingivitis, caries, ) (OD7). From Union health and family welfare center management of chronic otitis media (OD5) cases are referred in Figure 10.

The World Health Organization suggests that there are five core generic attributes that transcend the boundaries of specific disciplines and that should be present in everyone who cares for people with long term conditions [10]. These are patient centered care, partnering, quality improvement, information and communication technology and a public health perspective.

\section{Conclusion}

Non-professionally qualified having pre-existing medical training, receive top-up training which can be very short, although the length of training for those who will take on generalist roles is about one year. People who enter directly into the category receive training that ranges from 2 to 4 years and in most cases, undertake some kind of supervised clerical placement where they hone their skills; this may be during their training or afterwards in the form of a supervised internship. For many common conditions an effective, cost-efficient interventions would able to exist, if more widely implemented.

\section{References}

1. WHO (2006) Global shortage of health workers and its impact.

2. Ahmed SM, Hossain AMd, Chowdhury RAM, Bhuiya AU (2011) The health workforce crisis in Bangladesh: shortage, inappropriate skill-mix and inequitable distribution. Human Resources for Health 9: 1-7.

3. https://elibrary.worldbank.org/doi/abs/10.1596/978-1-4648-0536-3

4. Rapid review of international evidence Report developed by The Evidence Centre for Skills for Health. Key changes in the Healthcare workforce.

5. Zurn P, Dal Poz M, Stilwell B, Adams O (2002) Imbalances in the health workforce 2: 1-9.

6. ACHDHR (2007) A framework for collaborative Pan-Canadian health human resources planning. Ottawa: Health Canada, Advisory Committee on Health Delivery and Human Resources, HHR Planning Subcommittee.

7. Dussault G, Dubois CA (2003) Human resources for health policies: a critical component in health policies. Human Resources for Health 1: 1-16.

8. Singh D (2005) Which staff improve care for people with long term conditions? A rapid review of the literature. Birmingham: University of Birmingham, Health Services Management Centre.

9. Wagner $\mathrm{EH}$ (2000) The role of patient care teams in chronic disease management. BMJ 320: 569-572.

10. Sommers LS, Marton KI, Barbaccia JC, Randolph J (2000) Physician, nurse and social worker collaboration in primary care for chronically ill seniors. Arch Intern Med 160: 1825-1833. 